\title{
The Use of Acupuncture in Eye Diseases: An Overview
}

\author{
Xibin Liang ${ }^{1 *}$, Jingjun Wang² and Keshav Narain ${ }^{3 *}$ \\ ${ }^{1}$ Department of Neurosurgery, Stanford University, USA \\ ${ }^{2}$ Outpatient Unit, The First Hospital of Jinan City, China
}

${ }^{3}$ South Bay Retina, USA

*Corresponding author: Xibin Liang, Department of Neurosurgery, Stanford University, Stanford, CA 94305, USA

Keshav Narain, South Bay Retina, Inc. 455 0’Connor Dr. \#310, San Jose, CA 95128, USA

\section{Introduction (Historical overview)}

Allopathic or western medical approaches to ocular conditions such as cataract, diabetic retinopathy, and wet age-related macular degeneration have been largely successful, resulting in the decline of severe visual loss worldwide for patients with these conditions. Despite these successes, a variety of other ocular conditions continue to pose substantial ongoing threats to vision. Dry macular degeneration, optic nerve disease, and myopic degeneration not only lack effective treatments, but are increasing sharply. As the limits of western approaches become apparent, there is rising interest in alternatives. Traditional Chinese acupuncture has existed for thousands of years. The National Institutes of Health (NIH) now has a division dedicated to the investigation of alternative and complementary therapies. (https://www.nccih.nih.gov/). Clinicians should be familiar with existing knowledge and potential benefits of complementary and alternative therapies. Few western or allopathically trained clinicians are familiar with acupuncture. No consensus has been reached on the precise biomedical model for the mechanism of action of acupuncture. Neuromodulation, alteration in local blood flow, and micro-injury with repair have been proposed as factors involved in response to acupuncture.

The early record of acupuncture in the treatment of eye disease can be found in "The Yellow Emperor's canon of internal medicine" written in the Han dynasty of 2000 years ago. In the "Chapter Heat Disease of Ling Shu" $[1,2]$ it stated that "if redness and pain of the eyes start from the inner can thus, Yin Qiao should be taken as the needling point". In "Chapter the evil Qi and the morphology of Zang and Fu", it said "the Qi and blood from the 12 meridians and 365 branches go to the head and then to each orifice, the most essential part of it, the pure Yang Qi, goes to eyes and makes the eye clear", emphasizing a close relationship between the eye and the internal organs as well as other part of the body, which was thought to provide a basis of theory for acupuncture treatment of eye diseases [3]. From then on, acupuncture practitioners of different dynasties have contributed a variety of theories and treatment modalities, which tremendously enriched our knowledge of acupuncture treatment for eye diseases. Nowadays, more and more patients with eye diseases seek acupuncture treatment in China and around the world [4-6]. Ophthalmologists from Hong Kong, China and Sofia, Bulgaria published two earliest English case reports respectively in 1980s. In the first report, over 500 cases treated shows that acupuncture can be successful in the treatment of eye diseases, especially in cases of retinitis pigmentosa (RP), high myopia, cataracts, surgical aphakia, controlled glaucoma and re-attached ablation retinae [7]. In the second report, acupuncture treatments for myopia, glaucoma, RP and optic nerve atrophy showed some improvement in visual acuity subjectively [8]. A survey conducted in 2006-07 in US showed that 42 per cent of retinitis pigmentosa patients had tried acupuncture [9]. Here we review the recently published use of acupuncture as a primary and adjunctive treatment for ocular disease. We focus on glaucoma, optic atrophy, $\mathrm{RP}$ and amblyopia as they were the most common eye disorders presenting for treatment to acupuncturists.

\section{Clinical Studies}

\section{Glaucoma}

Despite progress in the development of novel medical and surgical approaches for treatment, glaucoma is still one of the leading causes of irreversible blindness worldwide [10]. Therefore, many patients sought acupuncture treatment to reduce progression 
of glaucoma. Wong and Ching [7] show a subset of patients with glaucoma showed improvement in visual acuity with three out of eight patients showing a decrease in intra-ocular pressure (IOP). A similar result was also shown in Liu's clinical study [8]. In 2004, Ren and Wang [11-13] summarized the reports of treatment of glaucoma with acupuncture and other acupuncture related treatment modalities published in China from as early as 1963. The reported modalities included body acupuncture (acupoints around the eye and in the other parts of the body, especially the foot, leg and hand), moxibustion, ear acupuncture, blood pricking, etc. Their conclusion was that acupuncture could reduce the intraocular pressure (IOP) and was effective in the treatment of glaucoma, especially for the open angle glaucoma. As with pharmacologic therapy, benefits were not permanent and failed to show lasting impact with respect to IOP reduction.

In a 2005 Japanese study, 11 glaucoma patients were given 9 sessions of acupuncture in 5 weeks. IOP was significantly improved at 15 minutes after acupuncture, and also at one week, two weeks and five weeks after acupuncture. Uncorrected visual acuity was improved at three to five weeks, and best-corrected visual acuity was improved only at five weeks. The acupuncture induced improvement in IOP disappeared four weeks after stopping acupuncture treatment [14].

In another study, hemodynamic changes in retrobulbar vessels of OAG eyes before and after acupuncture showed a significant decrease in the vascular resistance of the short posterior ciliary arteries along with the a decrease in IOP, suggesting an increase in retinal and choroidal blood flow after acupuncture $[15,16]$. Similarly, IOP decreases were also seen by Her et al. [17] and Yeh et al [18]. In Leszczynska et al's randomized study [19] Primary Open-Angle Glaucoma patients were randomly assigned either to an eye-specific acupuncture (group $\mathrm{I}, \mathrm{n}=28$ ) or to a non-specific acupuncture treatment (group II, n=28). Blood flow parameters were measured before and 10 minutes after treatment. They did not find the change of IOP but found that ocular blood flow increased significantly after the eye-specific acupuncture treatment. The inconsistent results of the above studies are likely due to numerous factors, including acupoint selection, needling technique, measuring method, subject recruitment, diagnostic criterion and medication history. The relationship between IOP and ocular hemodynamics has been shown to depend on arterial blood pressure (BP) and blood flow autoregulation (AR) [20-23], suggesting that the ability of IOP to induce noticeable changes in retinal hemodynamics depends on the levels of BP and AR of the individual. Thus, the impact of IOP reduction depends on hemodynamic criteria. Without controlling for those variables, it is difficult to show clear benefits of acupuncture upon glaucoma progression. Strong clinical evidence to justify the routine use of acupuncture is still lacking. Authors conclude that it is impossible to draw reliable conclusions from available data to support the use of acupuncture for treatment of patients with glaucoma $[24,25]$.

\section{Optic atrophy (Optic Neuropathy)}

The term optic atrophy indicates the end stage of a neuropathic process in which there is end stage damage to the optic nerve. Examples of optic atrophy include end stage glaucoma, nerves damaged from compression via tumors, etc. There are two metaanalysis studies regarding the effect of acupuncture in the treatment of optic atrophy. The first one Included 13 randomized clinical trials (RCT) studies of total 1180 eyes (acupuncture 619, control 561) [26]. Among these trials, two studies comparing manual acupuncture (as treatment) with medication alone (as control), 11 studies comparing manual acupuncture plus medication (as treatment) and medication alone (as control). Meta-analyses showed that the effect of acupuncture or combined with medicine was superior to medicine alone in terms of total effectiveness, visual acuity, and visual field, The authors concluded that acupuncture is superior to medicine in terms of improved visual acuity, visual field and pattern visual evoked potential. But they also mentioned that the flaw in the design of the clinical studies and a large scale, multiple-center, high-quality studies is needed to make stronger evidence. The second one included 9 studies (a total of 513 participants: 262 in experiment groups and 251 in control groups) [27]. Among three trials, three studies comparing manual acupuncture (as treatment) with medication alone (as control); six studies comparing manual acupuncture plus medication (as treatment) and medication alone (as control). The meta-analysis showed significant differences in favor of manual acupuncture or manual acupuncture plus medication compared with medication alone in the improvement of visual acuity, mean sensitivity of visual field, the latent period and the total effectiveness. However, due to serious methodological flaws in study design, such as discrepancy in randomization, lack of blindness, publication bias (all published in Chinese), and lack of follow-ups, etc. The authors failed to conclude that manual acupuncture is more effective than medicine alone.

\section{Retinitis pigmentosa (RP)}

Currently, there is treatment for only one of several thousand variants of retinitis pigmentosa. Thus, the vast majority of patients with this condition have no proven therapeutic options to improve or halt the slowly progressive visual loss. In Kiser AK, Dagnelie G 's survey, 42 per cent of RP patients tried acupuncture. A total of 96 patients participated in the survey and 61 per cent indicated a subjective improvement in vision. Kiser and Dagnelie [9]. To investigate the effect of acupuncture in RP, physicians from Wilmer Eye Institute, Johns Hopkins University in Maryland, and College of Optometry, Nova Southeastern University in Florida, conducted two clinical studies. In one study [28], 12 adult RP patients were given 10 half-hour sessions of acupuncture treatment over two 
weeks. Patients were administered pre- and post-treatment visual acuity, contrast sensitivity, visual fields, and dark-adapted full-field stimulus threshold testing (FST). Six of 12 subjects had significant visual function improvements after treatment. Three of nine subjects tested with the FST had a significant (13- to 53-fold) improvement in both eyes at one week after acupuncture, maintained for at least 10 to 12 months. Dark-daptation was shortened in both subjects tested on average by 48.5 per cent by one week. Four of the five subjects with psychophysically measured scotopic sensitivity improvements reported subjective improvements in vision at night or in dark environments. One subject had 0.2 log MAR improvement in VA; another had $0.55 \log C \mathrm{~S}$ improvement. Another subject developed more than 20 per cent improvement in the area of the Goldmann visual fields.

In another study [29], twenty-one RP participants were randomized (1:1:1) to transcorneal electrical stimulation (TES), electro-acupuncture (EA) or inactive laser acupuncture (sham control). After treatment of 10 half-hour sessions over 2 weeks, a significant improvement in retrobulbar central retinal artery mean flow velocity for both the TES and electro-acupuncture groups were found when compared to sham controls. Electro-acupuncture subjects had significant $34 \%$ greater increases in retinal blood flow in the macular vessels when compared to sham controls $(\mathrm{p}=$ 0.008), There was a significant difference in the proportion of eyes that had improved visual function when comparing EA with sham ( $29 \%$ vs. $0 \%$ ), indicating that acupuncture stimulation had a better treatment effect.

\section{Amblyopia}

The use of acupuncture for the treatment of amblyopia treatment has become more and more common in recent 20 years [30]. In 2013, a non-Cochrane review examined the efficacy of acupuncture for amblyopia. The authors analyzed 14 RCTs $(2,662$ participants) out of 115 possible trials (most were published in Chinese). They found that the acupuncture group had higher rates of clinical improvements than the conventional treatment group [31]. Later on, two RCT reports on using acupuncture in amblyopia treatment were published in English journals. Zhao, et al reported the results of two groups of anisometropic amblyopes aged 7-12 years, one group received acupuncture while the other group (control groups) received $2 \mathrm{~h}$ of occlusion therapy. At 15 weeks, follow-up subjects in the acupuncture group were found to have significantly greater improvements in visual acuity in comparison to the control group (2.27 lines and 1.83 lines respectively) [32]. Lam et al. [33] reported the effects of acupuncture on anisometropic children aged 3-7-year-old, Participants were randomized to receive spectacles alone $(n=42)$ or spectacles + acupuncture $(n$ = 41) for 15 weeks. Using a randomized cross-over trial method, they found a greater improvement in visual acuity using refractive correction with acupuncture compared with refractive correction alone.

Although it is still controversial regarding the design of these RCTs as the set-up of sham acupuncture, the balance between the time used in acupuncture group and the control group, etc $[34,35]$, the clinical data so far seem to support a favorable effect of acupuncture treatment of amblyopia. The flaws in the design of the clinical trials need to be minimized in future and clinical trials involving more participants seem necessary.

It needs to mention that recently Vanzini and Gallamini [36], developed an ultra-low-light-intensity Laser acupuncture and they use the laser acupuncture for the treatment of 13 cases of anisometropic children of 3-11-year-old. The treatment was performed once a week with each session lasted $<15$ minutes. After 6 weeks, 11 out of 13 patients received remarkable improvement. They thought that laser acupuncture could provide similar, if not better, results to conventional acupuncture stimulation, but with higher patient compliance.

\section{Mechanism}

There are few studies investigating the mechanism of acupuncture in the treatment of eye disease. As mentioned above some clinical studies show that acupuncture may increase intraocular blood flow. There are some experimental studies showing that acupuncture could stimulate the vision cortex. Another factor may be promotion of photoreceptor cell survival through a pathway of neuroprotection or anti-apoptosis, etc.

\section{Acupuncture affect vision area in the brain}

Acupuncture has been shown, using fMRI, to improve blood flow in the visual cortex. A laser acupuncture at UB-67 was found to cause activation in the cuneus corresponding to Brodmann Area (BA) 18 and the medial occipital gyrus (BA 19) of the ipsilateral visual cortex while placebo stimulation did not show any activation [37]. The visual cortex was also activated by in some subjects during conventional or electro-acupuncture over four visionimplicated acupoints on the right foot [38]. However, one report also showed that electroacupuncture stimulation at point in the leg produced fMRI signal decreases in the occipital cortex, and there is no difference between vision related acupoint with an adjacent non-acupoint [39]. The inconsistence in the above studies might be related to the difference in the acu-points and needling method used in the study.

\section{Effect on photoreceptor cell survival or function}

Acupuncture is capable of inhibiting morphological changes of photoreceptor cells [40], and electrical stimulation is capable of ameliorating MNU-induced photoreceptor degeneration and rectifying abnormalities in the inner visual signal pathways [41]. 
This action may be through an indirect effect on photoreceptors through its modulation of secretion of several retinal neurotrophic factors and nerve growth factors, anti-apoptotic or antiendoplasmic reticulum (ER) stress pathway molecules as well as changes in the microenvironment. Acupuncture can raise the expression of Bcl-xl and brain-derived neurotrophic factor (BDNF) of retina, so as to prevent optic nerve damage caused by intraocular hypertension [42]. Daily sessions of low-frequency EA to rats during a critical developmental stage of retinal cell degeneration cause an increase of retinal nerve growth factor (NGF) and NGF high-affinity receptor (TrkA) expression; and increase of outer nuclear layer thickness; and enhanced visualization, suggesting that acupuncture treatment of RP might work through activation of some neurotrophic factors and their receptors in the retina [43]. A recently published review on the biology of acupuncture treatment of amblyopia also point out that acupuncture induced changes in the action of neurotransmitters in the visual system, promoted the secretion and synthesis of brain-derived neurotrophic factor, and stimulated the expression of genes related to visual plasticity [44]. In addition, Acupuncture treatment at acupoint GB20 or BL1 and GV16 respectively can reverse some pro-apoptotic molecules and ER stress pathway molecules generated in axotomized retina [45].

\section{Conclusion}

Since more and more patients of eye disorder are seeking acupuncture treatment, it is important to validate the effect of acupuncture in the treatment of these diseases. Up till now, there are several case reports and RCTs on the use of acupuncture to treat such disorders as glaucoma, optic atrophy, RP and amblyopia. Most of these reports tend to support that acupuncture possesses beneficial effect in the treatment of these disorders. However, since the bias that existed in these case reports and the flaws in the design and carrying out of these RCTs, it is still unable to validate that acupuncture is an effective choice for these eye patients. Therefore, more rigorous, multicenter randomized, double blind, placebo-controlled, clinical trial of acupuncture as a treatment in these eye disorders are needed in the future studies. Several clinical experiments showed that acupuncture could increase the retina blood flow, low IOP and change the fMRI signal in the vision cortex of the brain. However, inconsistent results existed owing to the disparity in acupoint selection, needling technique, measuring method, subject recruitment, diagnostic and evaluation criterion, etc. A unified standard need to be postulated in doing these clinical experiments so as to minimize the variation and inconsistence. It is promising that some animal studies showed that acupuncture could stimulate the release of endogenous neurotrophic factors such as NGF, BDNF, and regulate the gene related to the apoptosis and ER-stress pathway. However, these research were still at the primary stage. High quality, systemic and consistent research studies are needed to investigate the mechanism of acupuncture in the treatment of eye disorders.

\section{References}

1. Unschuld, Paul U (2016) Huang di nei jing ling shu: the ancient classic on needle therapy: the complete Chinese text with an annotated English translation.

2. 灵枢经热病《黄帝内经·灵枢》_热病第二十三_中医世家(2020) http:// www.zysj.com.cn/lilunshuji/huangdilingshu/101-3-23.html.

3. 灵枢经邪气脏腑病形, 《黄帝内经·灵枢》_邪气藏府病形第四_中医世 家." Retrieved July 15, 2020 (http://www.zysj.com.cn/lilunshuji/huang.

4. Dhaliwal, Deepinder K, Siwei Zhou, Sandeep S Samudre, Nathan J Lo, et al. (2019) Acupuncture and Dry Eye: Current Perspectives. A DoubleBlinded Randomized Controlled Trial and Review of the Literature. Clin Ophthalmol 13: 731-740.

5. Ji, Meiqi, Yali Qin, Yingxin Zi, Rui Wang, Huan Meng, et al. (2018) Acupuncture for Ophthalmoplegia: Protocol for a Systematic Review. Medicine 97(24): e11065.

6. Yao, Su-Fen, Jian-Hao Zhao, Zhen Mao, Li-Jia Chen, et al. (2010) Acupuncture treatment on ophthalmic diseases. Zhongguo Zhen Jiu=Chinese Acupuncture \& Moxibustion 30(12):1029-1031.

7. Wong S, R Ching (1980) The Use of Acupuncture in Ophthalmology. The American Journal of Chinese Medicine 8(1-2): 104-153.

8. Dabov S, G Goutoranov, R Ivanova, N Petkova (1985) Clinical Application of Acupuncture in Ophthalmology. Acupunct Electrother Res 10(1-2): 79-93.

9. Kiser, Ava K, Gislin Dagnelie (2008) Reported Effects of Non-Traditional Treatments and Complementary and Alternative Medicine by Retinitis Pigmentosa Patients. Clin Exp Optom 91(2):166-176.

10. Friedman, David S, Roger CW Wolfs, Benita J O'Colmain, Barbara E Klein, et al. (2004). Prevalence of Open-Angle Glaucoma among Adults in the United States. Archives of Ophthalmology (Chicago, Ill.: 1960) 122(4): 532-538.

11. Quigley HA, AT Broman (2006) The Number of People with Glaucoma Worldwide in 2010 and 2020. The British Journal of Ophthalmology 90(3): 262-267.

12. Liu, Wen, Guang Yang, Xiao-jing Zhao, Yang-guang Song, et al. (2011) Impact of acupuncture on $24 \mathrm{~h}$ intraocular pressure of glaucoma. Zhongguo Zhen Jiu=Chinese Acupuncture \& Moxibustion 31(6): 518-20.

13. Ren Yanru, Wang Jingbo, 王静波. “针尒治疗青光眼概况-《上海针尒杂 志》(2004)年04期-中国知网.” Retrieved July 15, 2020 (https://mall. cnki.net/magazine/Article/SHZJ200404032.htm).

14. Kurusu, Masayuki, Kei Watanabe, Toru Nakazawa, Takashi Seki, et al. (2005) Acupuncture for Patients with Glaucoma. Explore (New York, N.Y.) 1(5): 372-376.

15. Takayama, Shin, Takashi Seki, Toru Nakazawa, Naoko Aizawa, et al (2011) Short-Term Effects of Acupuncture on Open-Angle Glaucoma in Retrobulbar Circulation: Additional Therapy to Standard Medication. Evidence-Based Complementary and Alternative Medicine: ECAM 2011:157090.

16. Takayama, Shin, Masashi Watanabe, Hiroko Kusuyama, Satoru Nagase, et al. (2012) Evaluation of the Effects of Acupuncture on Blood Flow in Humans with Ultrasound Color Doppler Imaging. Evidence-Based Complementary and Alternative Medicine: ECAM 2012:513638.

17. Her Jiann-Shyan, Po-Len Liu, Neng-Chin Cheng, Hung-Chang Hung, Po-Hsun Huang, et al. (2010) Intraocular Pressure-Lowering Effect of Auricular Acupressure in Patients with Glaucoma: A Prospective, Single-Blinded, Randomized Controlled Trial. Journal of Alternative and Complementary Medicine (New York, N.Y.) 16(11): 1177-1184. 
18. Yeh, Tsui-Yun, Jen-Chien Lin, Chi-Feng Liu (2016) Effect of Transcutaneous Electrical Nerve Stimulation through Acupoints of Pucan (BL 61) and Shenmai (BL 62) on Intraocular Pressure in Patients with Glaucoma: A Randomized Controlled Trial. Journal of Traditional Chinese Medicine=Chung I Tsa Chih Ying Wen Pan 36(1): 51-56.

19. Leszczynska Anna, Lisa Ramm, Eberhard Spoerl, Lutz E Pillunat, Naim Terai (2018) The Short-Term Effect of Acupuncture on Different Ocular Blood Flow Parameters in Patients with Primary Open-Angle Glaucoma: A Randomized, Clinical Study. Clinical Ophthalmology (Auckland, N.Z.) 12: 1285-1291.

20. Caprioli, Joseph, Anne L Coleman, Blood Flow in Glaucoma Discussion (2010) Blood Pressure, Perfusion Pressure, and Glaucoma. American Journal of Ophthalmology 149(5): 704-712.

21. Galassi, Fernando, Barbara Giambene, Roberta Varriale (2011) Systemic Vascular Dysregulation and Retrobulbar Hemodynamics in NormalTension Glaucoma. Investigative Ophthalmology \& Visual Science 52(7): 4467-4471

22. Guidoboni, Giovanna, Alon Harris, Simone Cassani, Julia Arciero, et al. (2014) Intraocular Pressure, Blood Pressure, and Retinal Blood Flow Autoregulation: A Mathematical Model to Clarify Their Relationship and Clinical Relevance. Investigative Ophthalmology \& Visual Science 55(7): 4105-4118.

23. Leske M Cristina (2007) Open-Angle Glaucoma--an Epidemiologic Overview. Ophthalmic Epidemiology 14(4): 166-172.

24. Law, Simon K, Lin Wang, Tianjing Li (2020) Acupuncture for Glaucoma. The Cochrane Database of Systematic Reviews 2: CD006030.

25. Law Simon K, Starrie Lowe, Samuel M Law, JoAnn A Giaconi, Anne L Coleman, et al. (2015) Prospective Evaluation of Acupuncture as Treatment for Glaucoma. American Journal of Ophthalmology 160(2): 256-265.

26. Dai, Yanli, Ming Liu, Yixin Zhang, Shihui Wei, et al. (2013) Meta analysis of acupuncture in the treatment of optic atrophy. Zhong Nan Da Xue Xue Bao. Yi Xue Ban=Journal of Central South University. Medical Sciences 38(3):283-290

27. Zhi, Fang-Yuan, Jie Liu, Xiao-Peng Ma, Jue Hong, et al. (2019) Manual Acupuncture for Optic Atrophy: A Systematic Review and Meta-Analysis. Evidence-Based Complementary and Alternative Medicine: ECAM 2019:1735967

28. Bittner, Ava K, Jeffrey M Gould, Andy Rosenfarb, Collin Rozanski, et al. (2014) A Pilot Study of an Acupuncture Protocol to Improve Visual Function in Retinitis Pigmentosa Patients. Clinical \& Experimental Optometry 97(3): 240-247.

29. Bittner, Ava K, Kenneth Seger, Rachel Salveson, Samantha Kayser, et al. (2018) Randomized Controlled Trial of Electro-Stimulation Therapies to Modulate Retinal Blood Flow and Visual Function in Retinitis Pigmentosa. Acta Ophthalmologica 96(3): e366-e376.

30. Maconachie, Gail DE, Irene Gottlob (2015) The Challenges of Amblyopia Treatment. Biomedical Journal 38(6): 510-516.

31. Yan, Xingke, Tiantian Zhu, Chongbing Ma, Anguo Liu, et al. (2013) A Meta-Analysis of Randomized Controlled Trials on Acupuncture for Amblyopia. Evidence-Based Complementary and Alternative Medicine: ECAM 2013:648054
32. Zhao, Jianhao, Dennis SC Lam, Li Jia Chen, Yunxiu Wang, et al. (2010) Randomized Controlled Trial of Patching vs Acupuncture for Anisometropic Amblyopia in Children Aged 7 to 12 Years. Archives of Ophthalmology (Chicago, Ill.: 1960) 128(12):1510-1517.

33. Lam, Dennis SC, Jianhao Zhao, Li Jia Chen, Yunxiu Wang, et al. (2011) Adjunctive Effect of Acupuncture to Refractive Correction on Anisometropic Amblyopia: One-Year Results of a Randomized Crossover Trial. Ophthalmology 118(8): 1501-1511.

34. Metheny, Jim (2011) Acupuncture and the Placebo Effect. Archives of Ophthalmology (Chicago, Ill.: 1960), 129(8): 1107-1108.

35. Milsky, Eliot (2011) Unfair Comparison of In-Office Acupuncture vs atHome Patching for Amblyopia. Archives of Ophthalmology (Chicago, Ill.: 1960) 129(7):963-964.

36. Vanzini, Marzio, Michele Gallamini (2016) Amblyopia: Can Laser Acupuncture Be an Option? Journal of Acupuncture and Meridian Studies 9(5): 267-274.

37. Siedentopf, Christian M, Stefan M Golaszewski, Felix M Mottaghy, Christian C Ruff, et al. (2002) Functional Magnetic Resonance Imaging Detects Activation of the Visual Association Cortex during Laser Acupuncture of the Foot in Humans. Neuroscience Letters 327(1): 5356.

38. Li Geng, Raymond TF Cheung, Qi-Yuan Ma, Edward S Yang (2003) Visual Cortical Activations on FMRI upon Stimulation of the Vision-Implicated Acupoints. Neuroreport 14(5): 669-673.

39. Kong, Jian, Ted J Kaptchuk, Julia Megan Webb, Jiang-Ti Kong, Yuka Sasaki, et al. (2009) Functional Neuroanatomical Investigation of Vision-Related Acupuncture Point Specificity--a Multisession FMRI Study. Human Brain Mapping 30(1): 38-46.

40. Ma Ruiling, Genchen Wu, Ren Zhang (2015) Effects of acupuncture on morphological changes of photoreceptor cells in rats with retinitis pigmentosa. Zhongguo Zhen Jiu=Chinese Acupuncture \& Moxibustion 35(11): 1149-1153.

41. Tao Ye, Tao Chen, Zhong-Yu Liu, Li-Qiang Wang, Wei-Wei Xu, et al. (2016) Topographic Quantification of the Transcorneal Electrical Stimulation (TES)-Induced Protective Effects on N-Methyl-N-Nitrosourea-Treated Retinas. Investigative Ophthalmology \& Visual Science 57(11): 46144624.

42. Sun He, Hui Zhang, Bai-song Lin (2010) Effect of acupuncture on the expression of Bcl-xl and BDNF of retina in rabbits with chronic intraocular hypertension. Zhongguo Zhen Jiu=Chinese Acupuncture \& Moxibustion 30(8): 661-664.

43. Pagani, Lucia, Luigi Manni, Luigi Aloe (2006) Effects of Electroacupuncture on Retinal Nerve Growth Factor and Brain-Derived Neurotrophic Factor Expression in a Rat Model of RetinitisPigmentosa. Brain Research 1092(1): 198-206.

44. Cao, Zhao-Xia, An-Guo Liu, Tian-Tian Zhu, Xiao-Juan Li, et al. (2018) Molecular Biology of the Treatment of Amblyopia with Acupuncture: A Review of Recent Research. Zhen Ci Yan Jiu=Acupuncture Research 43(3): 189-193.

45. Chen Jie, Li Zhang, Lanying Liu, Xueqin Yang, Fengzhi Wu, et al. (2019) Acupuncture Treatment Reverses Retinal Gene Expression Induced by Optic Nerve Injury via RNA Sequencing Analysis. Frontiers in Integrative Neuroscience 13:59. 


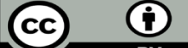

This work is licensed under Creative Commons Attribution 4.0 License

To Submit Your Article Click Here:

Submit Article

DOI: 10.32474/OAJCAM.2020.02.000146

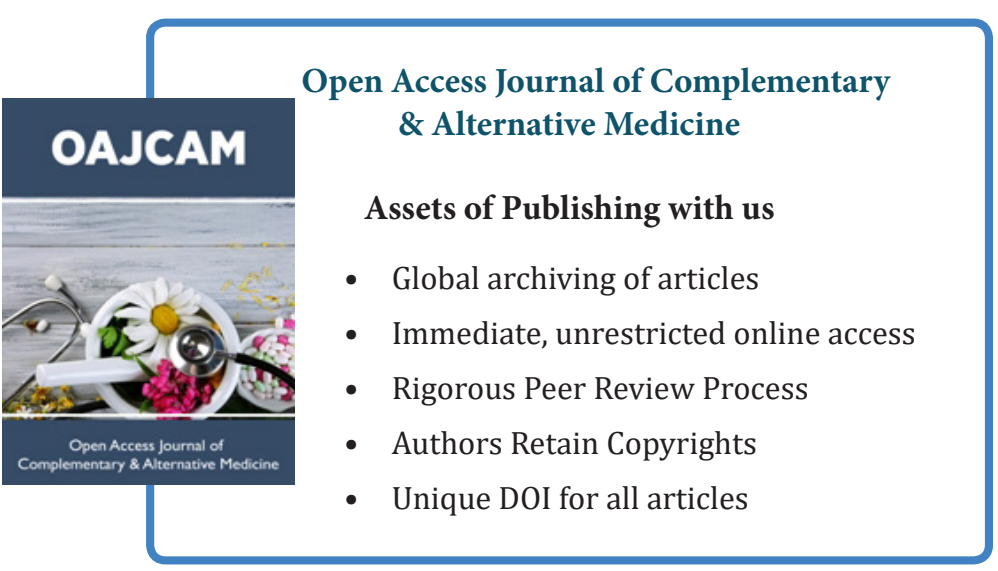

